\title{
Exercise and Schizophrenia
}

\author{
J. Velosa 1 , R. Avelar ${ }^{1}$, A. Delgado', F. Passos ${ }^{1}$, A. Grilo' 1 , M. Heitor ${ }^{1}$ \\ ${ }^{1}$ Hospital Beatriz Ângelo, Psychiatry Department, Loures, Portugal
}

\section{Objectives:}

To review the most recent evidence about physical interventions in patients with Schizophrenia

\section{Background and Aims:}

Patients with Schizophrenia have higher prevalence of metabolic syndrome than general population, which could be due to biological factors, antipsychotic treatment and also to decreased physical activity ${ }^{1}$.

Another issue is that antipsychotics have higher success in treating positive than negative symptoms. An example of the latter is cognitive dysfunction, which includes reduced short-term memory, slow processing speed and poor comprehension of social situations ${ }^{2}$. These symptoms, present in 50-90\% of the patients with a first episode psychosis and persisting in $20-40 \%$ of the cases, increase disease burden and problematic social outcome, namely a smaller social network and lower social functioning ${ }^{3}$.

Several different interventions such as Behavioral Therapy and Transcranial Magnetic Stimulation have been investigated but they did not lead to clinically relevant improvements ${ }^{4,5}$.

Recently, there's a growing interest in studying the influence of physical therapy approaches in those referred symptoms.

\section{Materials and Methods:}

A selective literature review, conducted on PubMed with the following keywords: "Exercise"; "Schizophrenia"; "aerobic", "anaerobic", "metabolic syndrome". Articles published in English were reviewed.

\section{Results:}

Regular physical activity - aerobic, strength exercises and yoga - have been shown to improve metabolic syndrome parameters, cognitive deficits, positive and negative symptoms, quality of life and global functioning ${ }^{1}$.

The impact in mental health depends more on the amount of exercise applied than the chosen modality ${ }^{6}$. This is corroborated by a meta-analysis ${ }^{7}$, where authors found that higher weekly duration tends to be associated with greater improvement in cognition as well as supervision from physical activity instructors.

Exercise also seems to be associated with brain volume changes in certain regions, particularly the hippocampus, a region known to be affected in Schizophrenia patients, with imagiological findings showing a smaller hippocampal volume. This has been correlated to deficits in cognition, memory and executive functioning ${ }^{8}$.

In addition, in these patients, serum brain-derived neurotrophic factor (BDNF) levels were shown to be significantly lower than in healthy controls and were associated with cognitive impairment ${ }^{9}$. Peripheral BDNF was shown to increase after aerobic exercise demonstrating a positive relation between BDNF and cognitive function, providing an important clinical link to enhanced neuroplasticity ${ }^{10}$.

\begin{tabular}{|c|c|c|}
\hline Schizophrenia & Impairments & Exercise \\
\hline$\uparrow$ & Negative Symptoms & $\downarrow$ \\
\hline$\downarrow$ & Working memory & $\boldsymbol{\uparrow}$ \\
\hline$\downarrow$ & Attention/Vigilance & $\boldsymbol{\uparrow}$ \\
\hline$\downarrow$ & Global functioning & $\uparrow$ \\
\hline$\downarrow$ & Quality of Life & $\uparrow$ \\
\hline
\end{tabular}
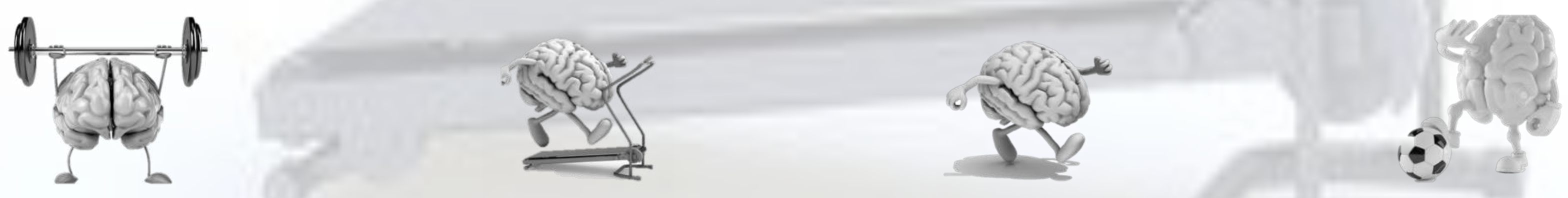

\section{Conclusions}

Exercise improves physical and mental health in patients with Schizophrenia, particularly cardiometabolic risk - thus reducing premature mortality -, social cognition, working memory and attention.

This is an important area for future research to complement functional recovery of patients.

References: 1- Schmitt, A. et al (2018) Effects of Aerobic Exercise on Metabolic Syndrome, Cardiorespiratory Fitness, and Symptoms in Schizophrenia Include Decreased Mortality. Frontiers in Psychiatry. 9. 690. 2- Falkai, P. (2017). Aerobic exercise and its effects on cognition in schizophrenia. Current Opinion in Psychiatry. 30. 1. 3- Degnan, A. et al (2018). Social networks and symptomatic and functional outcomes in schizophrenia: a systematic review and meta-analysis Soc. Psychiatry Psychiatr. Epidemiol. 53 (9), 873-888. 4- Aleman, A. et al 2017. Treatment of negative symptoms: where do we stand, and where do we go? Schizophr. Res. 186, 55-62. 5- Aleman, A. et al (2018). Moderate effects of noninvasive brain stimulation of the frontal cortex for improving negative symptoms in schizophrenia: metaanalysis of controlled trials. Neurosci. Biobehav. Rev. 89, 111-118. 6- Pajonk FG et al. (2010) Hippocampal plasticity in response to exercise in schizophrenia. Arch Gen Psychiatry, 67:133-43. doi: 10.1001/ archgenpsychiatry.2009.193 7- Firth, J. (2016). Aerobic Exercise Improves Cognitive Functioning in People With Schizophrenia: A Systematic Review and Meta-Analysis. Schizophrenia Bulletin. 43, 546-556 8- Gur RE et al (2000). Temporolimbic volume reductions in schizophrenia. Archives of general Psychiatry; 57(8);769-75 9- Zhang XY et al (2012) Low BDNF is associated with cognitive impairment in chronic patients with schizophrenia. Psychopharmacology 222(2):277-284 10- Kimhy D et al (2015) The impact of aerobic exercise on brainderived neurotrophic factor and neurocognition in individuals with schizophrenia: a single-blind, randomized clinical trial. Schizophr Bull 41(4):859-868 